\title{
The upgrade of the LHCb trigger for Run III
}

\author{
Mark Whitehead ${ }^{* \dagger}$ \\ CERN \\ E-mail: mark.p.whiteheadecern.ch
}

The LHCb upgrade will take place in preparation for data taking in LHC Run III. An important aspect of this is the replacement of the hardware trigger by implementing a full software trigger system. The progress and plans towards this objective are presented, including studies relating to the tracking and reconstruction sequence and the trigger output bandwidth division.

The European Physical Society Conference on High Energy Physics

5-12 July, 2017

Venice

* Speaker.

${ }^{\dagger}$ On behalf of the LHCb collaboration. 


\section{Introduction}

The LHCb experiment will be upgraded in preparation for data taking during LHC Run III. The instantaneous luminosity will increase by a factor of five from $\mathscr{L}=4 \times 10^{32} \mathrm{~cm}^{-2} \mathrm{~s}^{-1}$ in Run I and Run II to $\mathscr{L}=2 \times 10^{33} \mathrm{~cm}^{-2} \mathrm{~s}^{-1}$ in Run III. To cope with this change in luminosity a new trigger paradigm will be adopted, with the current two-stage hardware plus software trigger being replaced by a fully software trigger. Figure 1 shows a comparison of the trigger strategies for (left) Run II and (right) Run III. Removal of the hardware trigger is expected to facilitate an increase in the

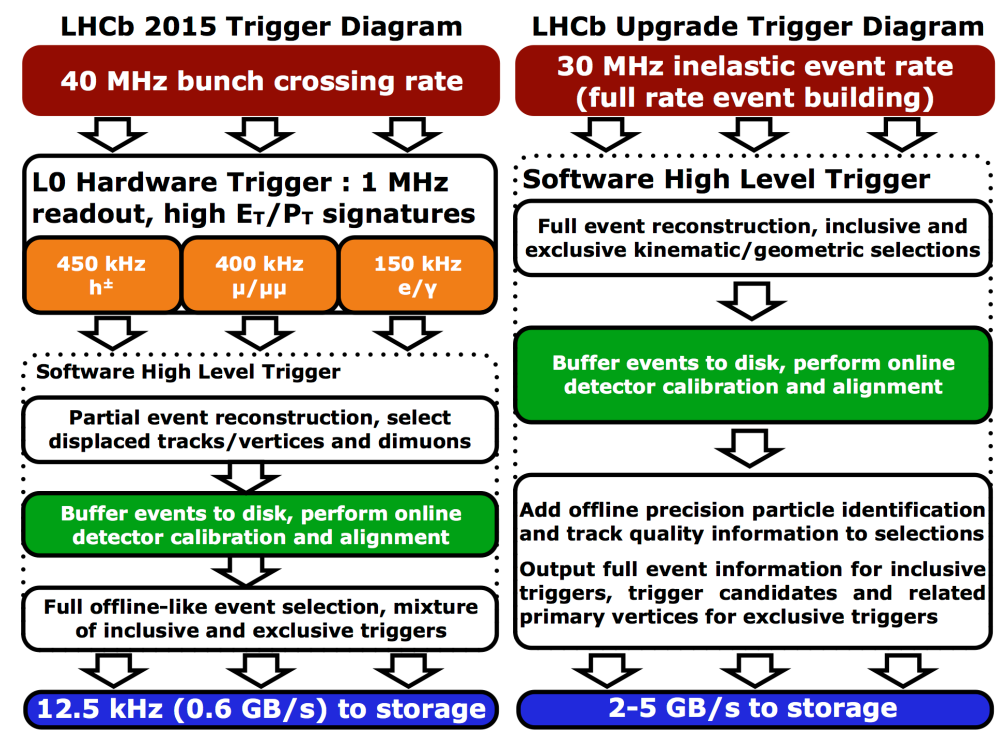

Figure 1: The LHCb trigger strategy in (left) Run II and (right) Run III.

trigger efficiency of purely hadronic decays modes by around a factor of two. It is also important to note that the challenges faced by the trigger system will also change, the rate of beauty and charm production mean that it is not sufficient to simply separate signal-like and background-like decay topologies. About $24 \%$ (2\%) of events will contain a reconstructible charm (beauty) hadron. Exclusive selections will be the standard, with some inclusive triggers remaining to preserve the breadth of the physics programme. To provide high purity samples with high efficiency these exclusive selections should be close to the final offline selection used in current analyses. Care must be taken with such advanced selections because the trigger will become more sensitive to detector performance effects, such as detection asymmetries, so the real-time alignment and calibration procedures, developed for LHC Run II, will become even more valuable.

\section{Tracking and reconstruction sequence}

For full details of the tracking and reconstruction sequences please see Ref. [1]. The strategy builds on that of LHCb in Run II (see Fig. 1), with the fast stage providing the reconstruction for the first part of the selection process, where the accepted candidates are buffered to disk. Then the real-time alignment and calibration tasks are executed before the best stage provides the full 
reconstruction. It is important to note that no further processing will be performed, the online best reconstruction will be of offline quality.

The performance of the fast stage is crucial for the upgrade trigger project. A first tuning of the algorithms, based on simulations of the expected Run III conditions, to optimise both speed and physics performance has been made. The environment in the upgrade era at LHCb is challenging; the average number of primary vertices per event is a factor of two or three times larger. Despite this, preliminary studies of the primary vertex resolution, shown in Fig. 2, look promising. In fact, the resolution in both (left) $x$ and (right) $z$ directions is better than for Run II. The fit function is given by

$$
\sigma(N)=\frac{A}{N^{B}}+C
$$

where $A, B$ and $C$ and free parameters and $N$ is the number of tracks associated to the vertex.
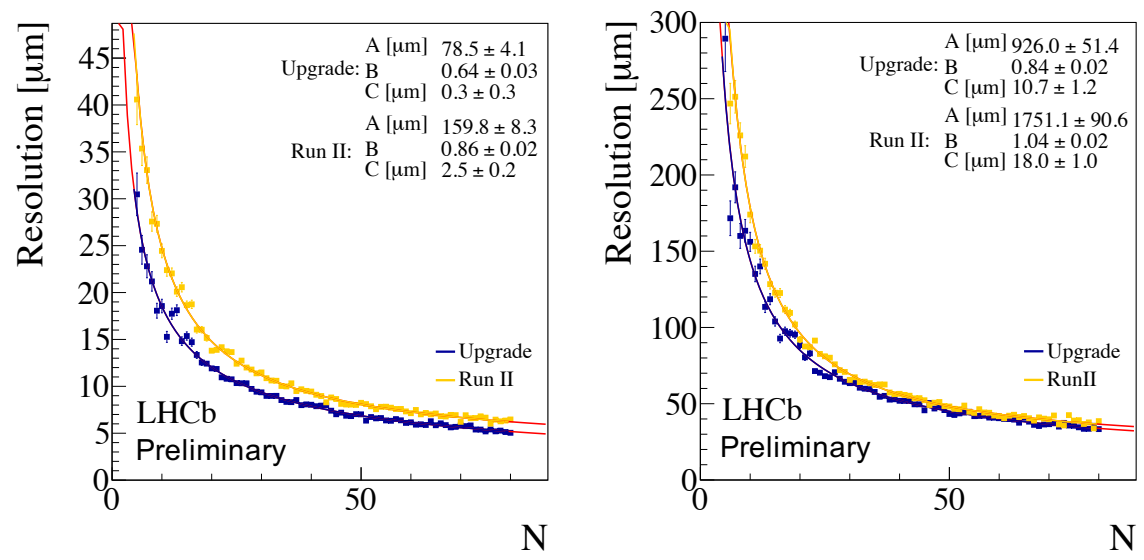

Figure 2: Primary vertex resolution in the (left) $x$ and (right) $z$ directions for the fast stage as a function of the number of tracks associated to the vertex. The fit function and fitted parameters are as described in the text. Figure reproduced from Ref. [1].

Studies of fake track (ghost) rejection, tracks reconstructed from pseudo-random combinations of hits, are also underway. Figure 3 shows the performance of a multivariate classifier designed to reject fake tracks applied to long tracks (tracks with hits in atleast the vertex locator and tracking stations downstream of the magent). Optimisation is in progress, but the latest training (red) is close to performing at the same level seen during Run II.

The timing and performance of the fast stage is in line with that expected from the upgrade trigger design report [2]. The timing per event is currently around $6 \mathrm{~ms}$, but this is expected to improve in the future. Tracking efficiencies at the fast stage are slightly better than expected, again with more improvements to come. Throughput performance targets are challenging to meet because the hardware performance growth at equal cost is slowing. Therefore a lot of effort is being spent to design and write new software that fully exploits the multiprocessor paradigm. A new computing technical design report is expected early next year. For more details on the timing and performances studies please see Ref. [1]. 


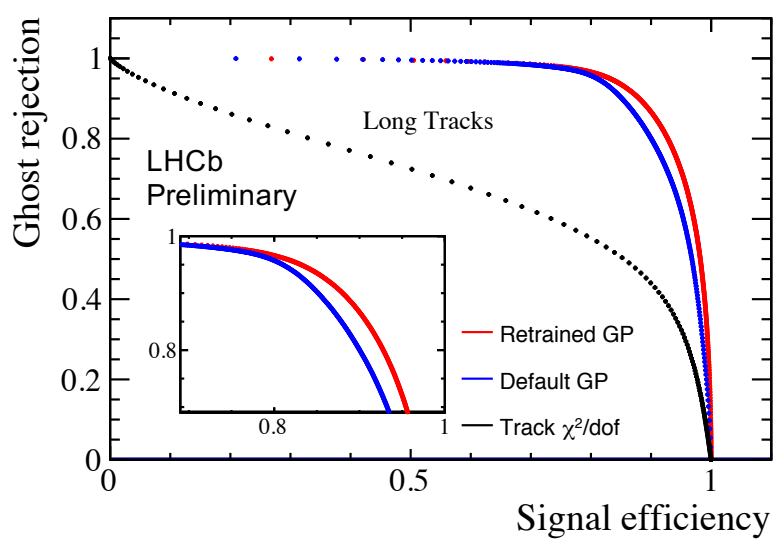

Figure 3: Comparison of fake track (ghost) rejection and signal efficiency, with (inset) a zoom of the optimal region, obtained with a multivariate classifier. Figure reproduced from Ref. [1].

\section{Trigger bandwidth division}

The division of the trigger output bandwidth, the data sample saved to offline storage, is another challenge for the upgrade trigger. Note that the total bandwidth available is limited by the available disk space rather than the network infrastructure or the trigger itself. Reduction of the event size to save more signal events in the same amount of disk space has already been used at $\mathrm{LHCb}$ for high rate channels [3]. This will become the standard approach in the upgrade era.

The proof of principle study documented in Ref. [4] uses an automated method to divide the output bandwidth between four charm decay modes. The bandwidth assigned per channel will ultimately be limited by the total number of channels and the physics priority decided by the collaboration. In this study a multivariate classifier is used to tune the output bandwidth consumed by each channel. A genetic algorithm is then used to assign the bandwidth per channel by minimising the following $\chi^{2}$ function by varying the classifier response for each channel

$$
\chi^{2}=\sum_{i}^{\text {channel }} w_{i} \times\left(1-\frac{\varepsilon_{i}}{\varepsilon_{i}^{\max }}\right) .
$$

Here $w_{i}$ is the channel weight for decay mode $i$ as decided by the collaboration (unity for each channel in this study), $\varepsilon_{i}$ is the signal efficiency at a given classifier requirement and $\varepsilon_{i}^{\max }$ is the maximum channel efficiency when it is allowed to consume the full available bandwidth. A penalty term is applied when the total available bandwidth is exceeded [4]. Signal efficiency and bandwidth are calculated from simulated samples. An example of the output from the bandwidth division algorithm is shown in Figure 4. Ultimately the signal efficiencies achieved will depend on the analysts, and their ability to define powerful selections using machine learning techniques.

\section{Summary}

Preparations for the upgrade of the LHCb trigger for Run III are well underway. The predicted performance of the tracking and reconstruction looks very promising on simulated data. The throughput performance will improve with further optimisation coming from significant work in 

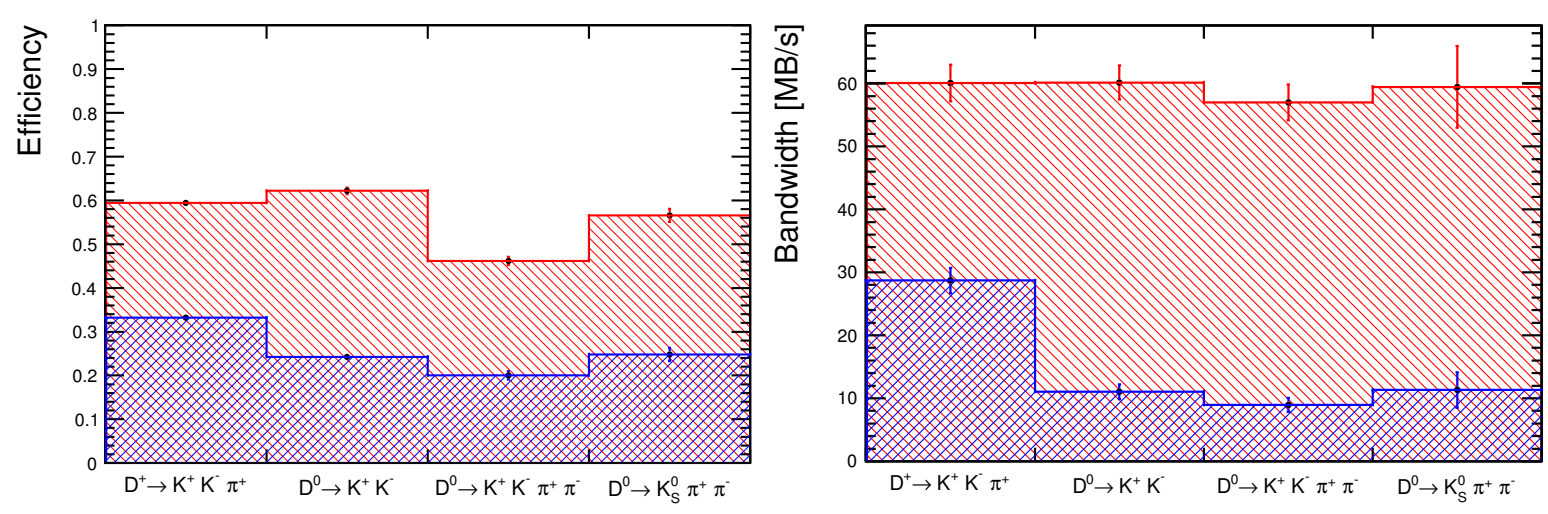

Figure 4: Example of the output of the bandwidth division studies for four charm decay modes, concerning (left) the signal efficiency and (right) the bandwidth usage. The red histogram shows the results when each channel is allowed to use all of the available bandwidth and the blue histogram shows the result of the division. The bandwidth limit is defined as $60 \mathrm{MB} / \mathrm{s}$. Figure reproduced from Ref. [4].

the coming years. First studies of the upgrade trigger bandwidth division have been performed as a proof of principle, with further studies extending this approach to cover the full physics programme to follow.

\section{References}

[1] R. Aaij et al., Upgrade trigger: Biannual performance update, LHCb-PUB-2017-005 (2017).

[2] R. Aaij et al., LHCb Trigger and Online Upgrade Technical Design Report, CERN-LHCC-2014-016 (2014).

[3] R. Aaij et al., Tesla: An application for real-time data analysis in High Energy Physics, Comput. Phys. Commun. 208, 35 (2016).

[4] C. Fitzpatrick et al., Upgrade trigger: Bandwidth strategy proposal, LHCb-PUB-2017-006 (2017). 